\title{
Studies on the Lysogenicity of Pseudomonas solanacearum with Special Reference to the New Type Phage Produced from a Doubly Lysogenic Strain
}

\author{
Mitsuo Tsujita and Chiaki Matsui
}

Received October 10, 1956.

UDC : 576. 851.1

We have reported previously that strain T-c200 is lysogenic (Tsujita, Matsui et a1. 1954), and that a doubly lysogenic strain can be prepared artificially by the infection of S-9 lysogenic bacteria with temperate T-c200 phage (Tsujita and Matsui 1955). From this doubly lysogenic strain new-types of phage which had host range characters of both parents were produced in addition to the parent types. On the basis of the experimental results obtained we have tried to explain this as a kind of prophage recombination. Since then we have done analytical experiments on this case of prophage recombination and arrived at an hypothesis unlike that proposed by Appleyard (1954). We propose that recombination takes place in the initial stage of double lysogenization of the singly lysogenic cells of strain S-9.

\section{Materials and Methods}

Bacterial strains :

1. P. solanacearum T-c200: a U.V. non-inducible lysogenic strain given by Prof. Norio Okabe (Faculty of Agriculture, Shizuoka University). By treatment with ultra-violet light the lysogenicity of the original strain can be removed. The symbol "T-c200\#" is given to the U.V. produced non-lysogenic T-c200 bacteria.

2. P. solanacearum S-9: a U.V. non-inducible lysogenic strain isolated from the strain given by Prof. Okabe.

3. P. solanacearum T-13, 4. P. solanacearum S-IX, 5. P. solanacearum E-79, 6, $P$. solanacearum E-3. Strains isolated from stocks given by Prof. Okabe.

7. $P$. solanacearum $\mathrm{T}-\mathrm{n} 30$ : a bacterial strain isolated from a wilt diseased tomato plant at the Agricultural Experiment Farm of Nagoya University in July 1955, it is non-lysogenic strain as judged when T-c200, S-9, S-IX and T-13 strains are used as indicators. The relation of this strain to the other bacterial strains is not clarified as yet.

Phages:

1. T-c200 phage: a temperate phage liberated from the T-200 strain; it has the ability to lysogenize T-c200\#.

2. T-c200 phage (v): a virulent mutant isolated from the $\mathrm{T}-\mathrm{c} 200$ strain.

3. S-9 phage: a temperate phage liberated from the S-9 strain. This phage is serologically related to the $\mathrm{T}-\mathrm{c} 200$ phage. 
4. S-9 phage (v): a virulent mutant isolated from $\mathrm{s}-9$ phage. 5. $\mathrm{Sp}_{1}$ : a virulent phage isolated by C. Matsui. This phage acts on strains " $\mathrm{s}$ " and S-9, but not on the T-c200 strain.

The following illustration shows the sensitivity of the bacterial strains to the various phages;

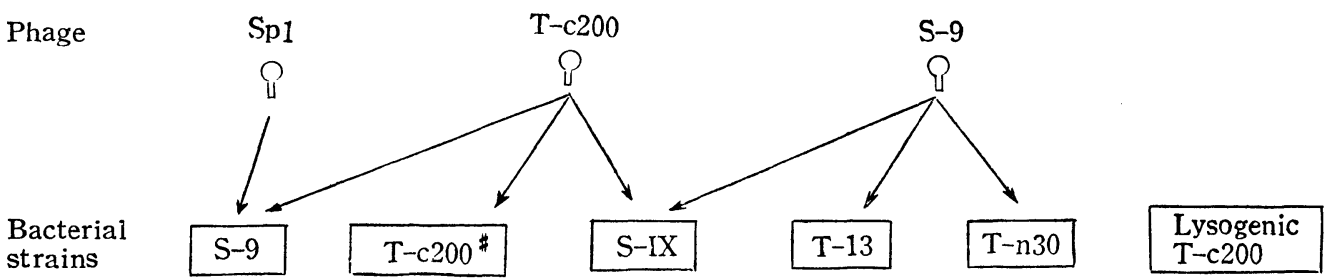

Fig. 1. Sensitivity of the bacterial strains to phage. An arrow indicates activity of phage against bacteria.

Preparation of phage suspensions: We have so far adopted for the multiplication of virulent phage the method of bacterial cultivation in liquid medium, which is generally used for the preparation of phage suspensions. In the case of temperate phage used in the present experiments, however, this method gave difficulty in getting final viral yield more than $10^{9} / \mathrm{ml}$. Therefore, in these experiments we have prepared high titer phage stocks according to Hershey's method (which had been described in "Method of study of bacterial viruses" published by M. H. Adams). As a host for the reproduction of T-c200 phage, T-c200\# bacteria were used; for S-9 phage, S-IX bacteria; for $\mathrm{Sp}_{1}$ phage, S-9 bacteria. Potato dextrose extract (P. D. E.) + glucose was used as a culture-medium.

\section{Experiment I}

S-9 bacteria were cultured for 24 hours in P. D. E. medium at $34^{\circ} \mathrm{C}$, then centrifuged and resuspended in P.D.E. medium. The superinfection was carried out at $34^{\circ} \mathrm{C}$ on $0.1 \mathrm{ml}$ of the bacteria by addition of $6.9 \mathrm{ml}$ of the $\mathrm{T}-\mathrm{c} 200$ phage. When a few minutes had elasped, the mixture was diluted 100 fold, and centrifuged twice (10,000 r.p.m. for 10 minutes) to remove the free phage. The number of non-infecting phage was determined in the supernatant of the first centrifugation.

After the centrifuged bacteria were suspended in $10 \mathrm{ml}$ of P.D.E. medium, they were used as a sample for the following experiments.

1. Bacterial colony count (1)

Bacterial colony counts were carried out by the agar plate method using $0.1 \mathrm{ml}$ of the sample; the temperature of incubation was $34^{\circ} \mathrm{C}$ and the counting was done after 48 hours. The colonies produced on the agar consisted of bacteria which showed a reversible response, lysogenized bacteria and non-infected bacteria. The lysogenized bacteria in this case include doubly lysogenic S-9 (T-c200) strain and 
a new strain producing $\mathrm{T}-\mathrm{c} 200$ phage only; the latter had probably originated from the substitution of the S-9 prophage with the superinfected temperate phage. The difference between bacterial concentration before phage infection and the bacterial colony count (1) indicates the number of lethal and productive (lysed) bacteria.

2. Bacterial colony count (2) selection.

After spreading $0.3 \mathrm{ml}\left(1.7 \times 10^{10} / \mathrm{ml}\right)$ of $\mathrm{Sp}_{1}$ phage solution over the surface of the agar, $0.1 \mathrm{ml}$ of the sample was also spread, and the plates were kept at $34^{\circ} \mathrm{C}$ for 48 hours when the colonies produced were counted. These consisted only of S-9 (T-c200) bacteria which had been lysogenized by T-c200 phage, i. e. S-9 bacteria which possessed $\mathrm{T}-\mathrm{c} 200$ prophage. The $\mathrm{Sp}_{1}$ phage resistant factor in the $\mathrm{T}-\mathrm{c} 200$ prophage is quite stable genetically, so when the S-9 lysogenic bacteria, which are inherently sensitive to $\mathrm{Sp}_{1}$ phage, are lysogenized by $\mathrm{T}-\mathrm{c} 200$ phage they invariably gain resistance to $\mathrm{Sp}_{1}$ phage. While the $\mathrm{S}-9$ colony may contain some bacteria which changed to $\mathrm{Sp}_{1}$ phage-resistance owing to spontaneous mutation, this rate may be considered to ke very small, any may be neglected. Thus the number of bacteria which showed a reversible response may be obtained by subtracting the bacterial colony count (2) and the number of non-infected bacteria from the bacterial colony count (1). When some bacteria are infected with single phage (multiplicity $\ll 1)$, as is the case, then the number of phage infected bacteria may be considered to follow the Poisson distribution. The number of non-infected bacteria may be obtained, therefore, without an appreciable error by subtracting the number of phage adsorbed from the initial number of bacteria present in the sample.

3. Plaque counts

One tenth $\mathrm{ml}$ of the sample was spread on a mixed indicator consisting of equal parts of $T-200 \#$ bacteria and $T-13$ bacteria (or $T-n 30$ bacteria), and $0.3 \mathrm{~m} 1$ of $\mathrm{Sp}_{1}$ phage suspension for the selection of bacteria which had T-c200 prophage. Then a plaque count was made. The results obtained in these experiments are shown in Table 1.

While, among the experimental results shown in Table 1, there are many phenomena to be discussed genetically and virologically, we would like to concentrate our attention on the phenomenon of the plaques producd on the mixed indicator.

\section{Working hypothesis}

In order to develop a clear plaque on the mixed indicator, consisting of T-c200\# bacteria and T-13 bacteria, both bacteria have to be lysed at the same time. When T-c200\# phage or S-9 phage are spread on the mixed indicator independently, a plaque is never produced because each of those phage only shows its affinity to either of the two bacterial strains independently. The fact that when S-9 bacteria were infected and lysogenized by $\mathrm{T}-\mathrm{c} 200$ phage they liberated phage capable of attacking both bacteria. T-c200\# and T-13 and of developing a plaque on the mixed indicator, will be discussed. We might consider the following four standpoints based 


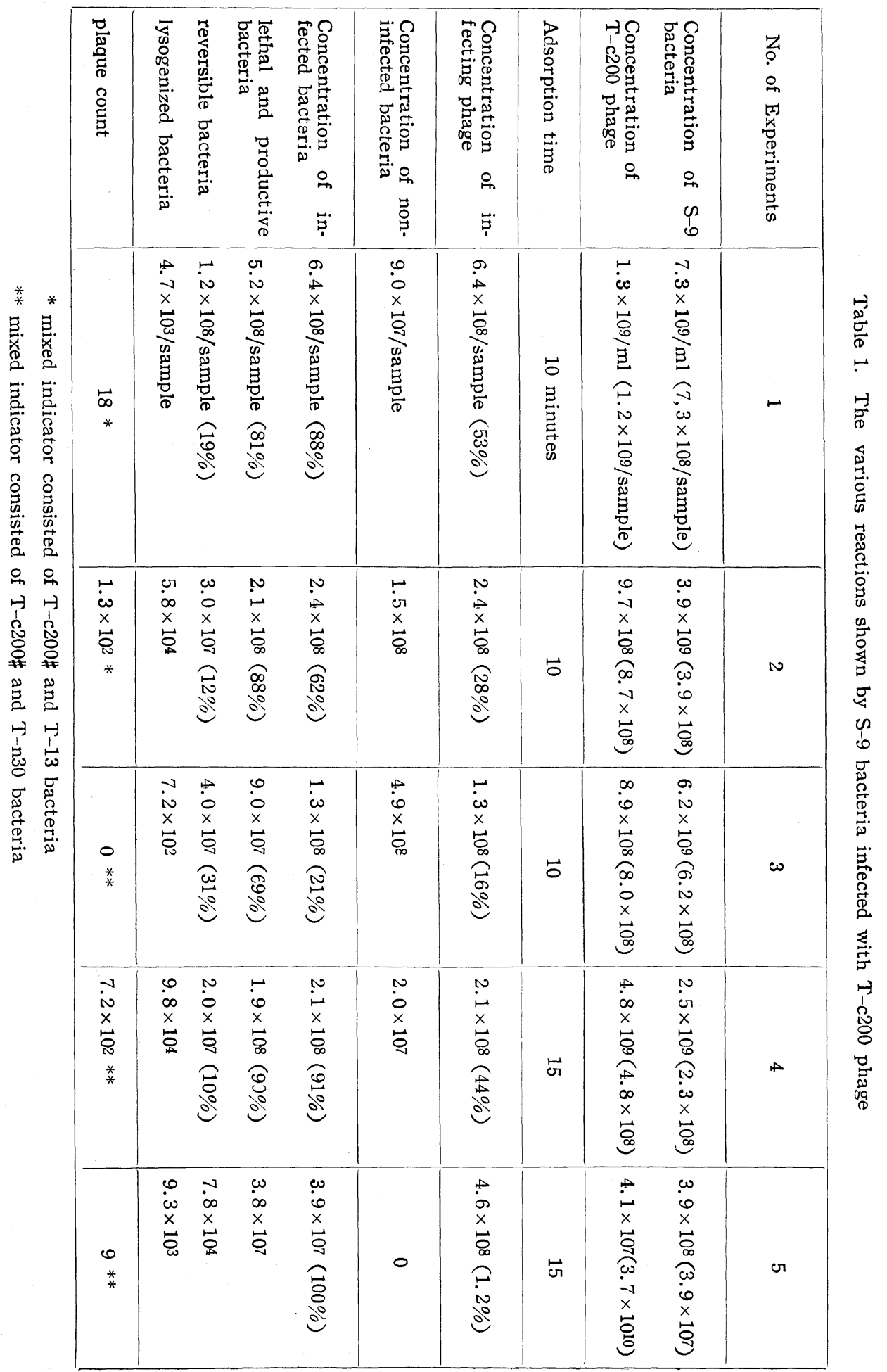


on various experimental results with virulent and temperate phages worked on by several investigators in the past.

1. Both phages produced by a doubly lysogenic bacterial cell.

If S-9 bacteria kecome double lysogenic by infection with T-c200 phage, and during the 1ytic cycle T-c200 and S-9 phages are matured and liberated at the same time, then T-c200 phage will attack the T-c200\# bacteria and S-9 phage will do the same on T-13 bacteria separately. As a result of the co-operative work of both phages, a plaque will be developed on the mixed indicator. Accordingly a plaque can be estimated as a mixed population of T-c200 phage and S-9 phage. If this is the case we may reasonably expect to isolate of T-c200 phage and S-9 phage from the same plaque. Moreover, in so far as the two re-isolated phages are not temporarily combined in some way, each of them can not produce a plaque when it is spread on the mixed indicator. However, the phage extracted from the plaque thus produced on the mixed indicator were respread on the mixed indicator and many plaques were obtained. This fact demonstrates the incorrectness of the above hypothesis in its simple form.

2. Host-induced modification

When phage multiplies in a new host, the newly produced phages are often subjected to an influence of the host cell and their host range specificity is modified. This is a temporary non-inheritable phenomenon, which is called host-induced modification (host controlled variation) in the case of T-phages of Escherichia coli (Bertani and Weigle 1953), the phage of Shigella dysenteriae (Nojima and Fukumi 1954 a, b, c), the phage of $P$. solanacearum described by Okabe (1953). Supposing that when T-c200 phage multiplies in S-9 bacterial cells, its host range becomes temporarily larger and it can attack T-13 bacteria though it is of the T-c200 genotype. Then plaques would be produced by the modified phage on the mixed indicator if growth on the T-13 host also modified the phage it produced. The phenomenon we observed can be judged not to be this kind of host-induced modification on the basis of the following experimental results.

When they are released from S-IX bacteria T-c200 phage does not show any affinity to T-13 bacteria. By lysogenization of S-IX bacteria with S-9 phage, singly lysogenic S-IX (S-9) bacteria can be obtained. When T-c200 phage multiplies in these bacteria they produce phage which can make plaques on the mixed indicator. This means that in order to extend this host range of the newly-produced phage, the existenece of S-9 prophage is required; there is no influence of the S-IX bacterium itself (Anderson 1955).

3. Spontaneous mutation.

As shown in the table, there is variation in the frequency of appearance of the new-type phage. There was only one experiment which produced no new-type phage; in the other 4 experiments we obtained a high frequency. In fact the frequency was too high to attribute to spontaneous mutation. Furthermore, plaque- 
forming bursts are produced by lysogenization of S-9 bacteria or S-IX (S-9) bacteria with T-c200 phage, but not by either S-9 bacteria or T-c200 bacteria alone. Consequently, it seems quite difficult to believe that the new-type phage arise by spontaneous mutation.

4. Recombination.

If recombination can occur between $\mathrm{T}-\mathrm{c} 200$ prophage and $\mathrm{S}-9$ prophage inside the body of S-9 bacterial cells infected with T-c200 phage, a part of the matured phage produced by the cells will be provided with characters peculiar to both phages. Thus one phage particle can attack and lyse either T-c200\# bacteria or T-13 bacteria and can produce a plaque on the mixed indicator. Besides, phage which have the same character as the parent phages can also be isolated from the plaque.

As we have said before, since both phages T-c200 and S-9, are at least necessary for producing plaques on the mixed indicator, and at the same time, a phage which is able to attack both bacterial strains can be isolated, this new-type phage can be considered as derived from recombination of T-c200 prophage and S-9 prophage. We will describe later the nature of this prophage recombination.

We have carried out the following experiments to see whether the above phenomenon might occur in other bacterial strains lysogenized with the same temperate phages.

\section{Experiment II}

In experiment $\mathrm{I}$, doubly lysogenic $\mathrm{S}-9(\mathrm{~T}-\mathrm{c} 200)$ was prepared by infecting $\mathrm{T}$ c200 phage on S-9 bacteria which are inherently lysogenic; identification of newtype phage liberated from them is made by observing the change of host range. In experiment II, we have examined whether there is production of new-type phage by making S-IX bacteria lysogenic with S-9 and T-c200 phage. In this case, after 5 day's culture of S-9 bacteria on P.D.E. medium, the bacterial suspension was centrifuged (10,000 r.p.m., 10 minutes) and the supernatant was filtered through a Chambeland filtration apparatus $\left(\mathrm{L}_{3}\right)$. The plaques produced by phages in the filtrate were enumerated using S-IX bacteria as an indicator. Some of the plaques produced on the layer of S-IX bacteria were clear while others were not. The latter were seeded on an agar plate spread with S-9 virulent phage $\left(2.9 \times 10^{9} / \mathrm{ml}\right)$ and colonies of bacteria which are immune to the phage were formed. By this treatment, S-IX bacteria lysogenized with S-9 phage, that is lysogenic S-IX (S--9) bacteria, can be selected. The ability of the lysogenic S-IX (S-9) bacteria to produce S-9 phage is a genetically stable character, and it cannot be lost by cultivation for many generations. On the other hand this phage shows an affinity for $\mathrm{T}-\mathrm{n} 30$ bacteria like the original S-9 phage, and does not attack T-c200\# bacteria. S-IX bacteria have no affinity for $\mathrm{Sp}_{1}$ phage (which are not even adsorbed), but lysogenic S-IX (S-9) bacteria changes to have affinity for $\mathrm{Sp}_{1}$ phage. A similar modification of affinity for $\mathrm{Sp}_{1}$ virulent phage could be observed in E-c79 and E-3 bacterial strains when 
they were lysogenized with the S-9 temperate phage. It was stated before that when S-9 bacteria, which are sensitive to $\mathrm{Sp}_{1}$ phage, are lysogenized with $\mathrm{T}-\mathrm{c} 200$ temperate phage they become genetically resistant to the same phage.

On the other hand, some biochemical characters can be changed by lysogenization of the normal cells with T-c200 temperate phage. Namely, the T-c200 cells fail to grow well on lactose and mannitol. When the normal cells which grow on the medium with lactose and mannitol are lysogenized with $\mathrm{T}-\mathrm{c} 200$ temperate phage they come poorly able to use these nutrients for their growth (Okabe 1955, Tsujita 1956). This fact suggest that on the $\mathrm{T}-\mathrm{c} 200$ phage chromosome several genes, homologous to the genes of bacterial chromosomes, are located, and that they are introduced with the prophage at the time of lysogenization

Thus it may be said that S-9 prophage or T-c200 prophage are able to become a genetic component of the bacterial cell by fixation on the bacterial chromosome and that they are able to change the affinity to phage of the host or some biochemical characters of the host cell. This phenomenon can be considered as a kind of transformation by phage, which is similar to the fact that the ability to produce toxin is related to lysogenesis in Corynebacterium diphtherias (Freeman 1951, Freeman and Morse 1952), or to the fact that the antigenic character is closely related to lysogenesis in Salmonela typhimurium (Iseki 1955, Terada 1956).

We have obtained the results shown in Table 2 using lysogenic S-IX (S-9) bacteria infected with $\mathrm{T}-\mathrm{c} 200$ phage in the same way as in experiment $\mathrm{I}$.

From the data shown in Table 2, it is suggested that a new type phage is produced when S-IX bacteria were made doubly lysogenic by S-9 phage and T-c200 phage. This is proved by replating the new-type phage from the plaque on the mixed indicator. Thus, we could demonstrate that the phenomena which were

Table 2. Various reactions shown by T-c200 phage infected S-IX (S-9) bacteria.

\begin{tabular}{|l|c|c|}
\hline Experiment number & \multicolumn{1}{|c|}{3} & \multicolumn{1}{|c|}{7} \\
\hline Concentration of S-IX (S-9) bacteria & $8.2 \times 109 /$ sample & $3.7 \times 10^{8}$ \\
\hline Concentration of T-c200 phage & $4.1 \times 109$ & $1.6 \times 10^{8}$ \\
\hline Adsorption time & 13 minutes & 15 minutes \\
\hline Concentration of infected phage & $3.7 \times 109(90 \%)$ & $9.7 \times 10^{7}(61 \%)$ \\
\hline Concentration of non-infected bacteria & $4.5 \times 109$ & $6.4 \times 10^{8}$ \\
\hline Concentration of infected bacteria & $4.1 \times 109(45 \%)$ & $9.7 \times 10^{7}(13 \%)$ \\
\hline Lethal and productive bacteria & $1.2 \times 109(30 \%)$ & $1.3 \times 107(13 \%)$ \\
Reversible bacteria & $2.7 \times 109(66 \%)$ & $7.3 \times 10^{7}(74 \%)$ \\
Lysogenized bacteria & $2.0 \times 108(4 \%)$ & $1.1 \times 10^{7}(12 \%)$ \\
\hline Plaque count* & $9.3 \times 102$ & $1.9 \times 10^{2}$ \\
\hline
\end{tabular}

* Mixed indicator consisted of $\mathrm{T}-\mathrm{c} 200 \#$ and $\mathrm{T}-\mathrm{n} 30$ bacteria. 
observed in experiment I could also occur when other bacterial strains than S-9 are used.

\section{On the type of recombination.}

There are two cases of formation of new-type phage by recombination which so far have been clarified in phage genetics. One case is the recombination of vegetative phage in the maturation stage of lytic cycle (Hershry 1953) and the other case is recombination at the stage of prophage which resides on the chromosome of the host (Appleyard 1954). In terms of this, the results obtained in our experiments will be analysed and discussed.

1. Recombination of vegetative phage: It is assumed that within the doubly lysogenic S-9 (T-c200) strain T-c200 prophage coexists with S-9 prophage in a paired form which attaches to the bacterial chromosome at a site which corresponds to a simple bacterial gene. When both prophages become vegetative phages in the lytic cycle they multiply and become mature phage through a maturation process. In this vegetative stage if genetic exchange occurs between the phages, recombinants will exist in the phage population which is newly produced. Accordingly, this recombination in the vegetative stage is not a genetic problem of the bacterial cell itself.

2. Recombination of prophage: If there exists a process formally similar to somatic crossing over by means of which the genes of the two related prophages carried in one cell can recombine, then new-type phage may be produced from such a cell. If the bacteria with recombinant prophages thus produced multiply themselves, then the ability to produced the new-type phage is inherited in successive generations and may become a hereditary character of the bacteria.

While the two types described above differ from each other in the stages in which recombination occurs, both prophages of T-c200 and S-9 have a stable stage of co-existence in the paired form. This state may be understood as the doubly lysogenic condition.

On the other hand, in our previous reports it was judged that the 1st type of recombination may not occur for the following reasons:

i) When doubly 1ysogenic cells enter the 1ytic cycle, multiplication of T-c200 phage is likely to exclude that of S-9 phage, since it seems that one or the other mature phage alone is produced from each of the cells. Recently to make clear this point an experiment was carried out. A small amount of the supernatant of a doubly lysogenic S-9 (T-c200) culture was spread on a single indicator S-IX and plaques produced were separately homogenized and the kinds of phages in each of the plaques were analysed. Among 48 plaques tested, 42 were formed by T-c200 phage only and the other 6 by S-9 phage only. No plaque produced by the cooperation of both phages was found. This result indicates that T-c200 and S-9 phages can not be simultaneously produced from a doubly lysogenic cell. 
ii) When virulent mutants of both phages infect sensitive bacteria, no new-type phage is obtainable by recombination in the vegetative phage.

From those facts, we have concluded that there is a suggestion of the existence of prophage recombination. Since then, we have carried out a series of experiments to examine the frequency of appearance of recombinants in clones of cells originating from a single cell on P. D. E. medium using the S-9 (T-c200) doubly lysogenic strain.

If the new-type phage are produced by the latter type of recombination according to the hypothesis produced by Appleyard, then the liberation of the new-type phage from clonal cells of the doubly lysogenic cell should be always expected. In our experiments, done more than 100 times up to the present, only S-9 phage and T-c200 phage could be isolated together from the medium in which clones of cell originating from one cell were cultured. This fact also disproves the possibility that the new-type phages were host modified variants.

As previously stated newly obtained lysogenic bacteria in the present experiment consisted of doubly 1ysogenic S-9 (T-c200) cells and of substituted cells producing T-c200 phage only. The latter cells as well as the new-type phage can be found only at the initial period of lysogenization of S-9 bacteria by T-c200 temperate phage. Hence, we could infer that a substitution of the S-9 prophage by the
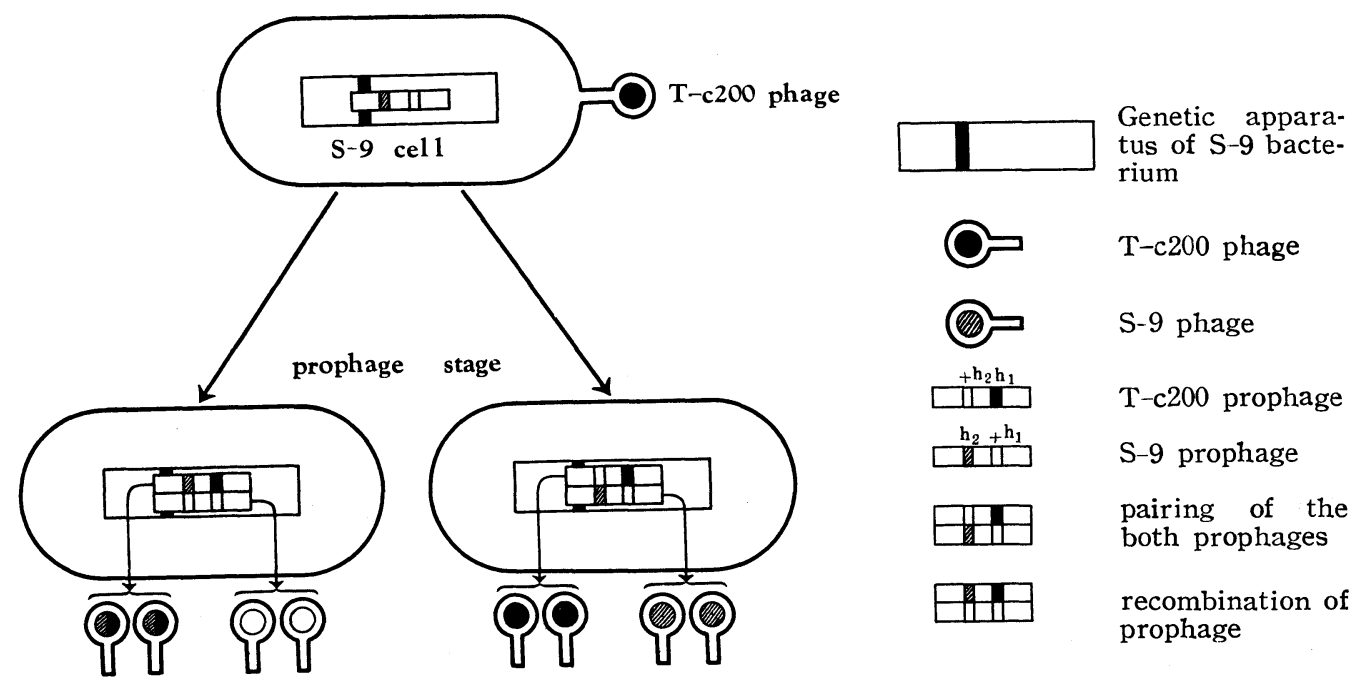

Fig. 2. Diagrammatic figure of the assumed recombination at the prophage stage.

superinfected $\mathrm{T}-\mathrm{c} 200$ prophage occurs at the initial period of 1ysogenization, and we might suggest a new mechanism of recombination in the prophage stage shown in Fig. 2. Namely when S-9 bacteria are infected with T-c200 phage and lysogenized, pairing occurs between the two prophages, $T-c 200$ and $S-9$, which reside on the chromosome of bacteria. Furthermore, in some bacterial cells, at the time of 
lysogenization, the substitution of the S-9 prophage with the superinfected T-c200 proghage occurs and is some other cells, at the same time, recombinant prophage are produced as the result of genetic exchange occuoring between the newly entered T-c200 prophage and the S-9 prophage. Thus, a bacterial strain able to produce the new-type phage appears.

Furthermore, when bacteria are picked up at random from the colony and seeded on the agar plate the strain which bears recombinat prophage cannot be easily isolated. Although this fact may be due to a low frequency of appearance of such a recombination further experiments is now carrying on to make clarify this point.

\section{Summary}

1. By the infection of S-9 bacteria with T-c200 prophage various responses, lethal productive reversible and lysogenic are observed. Some of doubly lysogenized S-9 (T-c200) bacteria liberate phage with a new-type of host range.

2. By infection with both S-9 and T-c200 phage, non-lysogenic S-IX bacteria can be changed into doubly lysogenic S-IX (S-9, T-c200) forms. In the doubly lysogenic bacterial strain thus formed, the new-type of phage is also produced.

3. When the doubly lysogenic bacteria are isolated it seems that they give rise to cells which produce only one or the other parent type phage. It is concluded that recombination occurs only at the time the doubly lysogenic state is initiated.

\section{Aknowledgement}

The authors wish to express their appreciation to Prof. Dr. Hirai for his encouragement and advices to this investigation, and to Prof. Okabe for kindly providing his valuable materials. They also wish to thank Prof. F. J. Ryan for the criticism of the manuscript as well as for his valuable assistance accorded in the preparation of the final manuscript.

\section{Literature cited}

Adams, M. H.. 1950, Methods of study of bacterial viruses in " Methods in medical Research" 2 The Year Book Publishers., Chicago.

Anderson, E. S., 1955, Consideration of the Vi-phage types of Salmonella typhi on a structural basis. Nature 175(4447): 171-173.

Appleyard, R. K., 1943, Segregation on new lysogenic types during growth of a doubly lysogenic strain derived from Esherichia coli $\mathrm{K} 12$. Genetics 39(4): 440-452.

Bertani G., and Weigle, J. J., 19:3, Host controlled variation in bacterial viruses. J. Bact. 65: $113-121$.

Freeman, V. J., 1951, Studies on the virulence of bacteriophage infected strains of Corynebacterium diphtheriae. J. Bact. 61 : 675-688.

Freeman, V. J., and Morse, I. U., 1952, Further observations on the change to virulence of bacteriophage infected avirulent strains of Corynebacterium diptheriae. J. Bact. 63 : 407-414.

Hershey. A. D., 1953, Inheritance in bacteriophage. Advances in genetics Vol. V, 89-106.

Iseki, S., and Sakai, T., 1953, Artificial transformation of antigens in Salmonella E group II. Antigen-Transforming factor in Bacilli of subgroup E2. Proc. Jap. Acad. 29: 129-131. 
Nojima, T., and Fukumi, H., 1954a, Host-controlled variation of Shigella bacteriophage. J. Med. Sci. \& Biol. 7 : 365-375.

1954b: Semi-resistance of bacteria to a bacteria to a bacteriophage. Second report on host-controlled variation. Ibid. 385-394

1954c, An essential feature of host range mutation. Third report of host-controlled variation. Ibid : $585-614$.

Okabe, N., and Goto, 1953, Studies on Pseud. solanacearum II. A comparative study of Pseud. solanacearum bacteriophages. Reports of Fac. of Agr. Shizuoka University, $3: 81-100$.

, 1955, Studies on Pseud. solanacearum X. Genetic change of the bacterial strains induced by temperate phage T-c200. Ibid. No. 5: 57-62.

Terada, M., 1956, Studies on bacterial viruses. Naya Publishing company, Ltd.

Tsujita, M., 1956, Lysogenic conversion of some genetic characters including sugar fermentation in Pseud. solanacearum. Abstract of lecture in the international genetics symposium (Japan).

Tsujita, M., and C., Matsui, 1955, A doubly lysogenic strain of Pseud. solanacearum. Proc. Japan Acad. 3(3) : 180-185.

Tsujita, M., Matsui, C., Tsuda, S., and Yoshizawa, 0., 1954, Studies on the lysogenicity of Pseud. solanacearum. 1 Lysogenic strain T-c200. Virus 4(4) :308-312. 1955, Ann. Rep. Nat. Inst. Genet. $5: 80-82$

Weigle, J. J. and Bertani, G., 1953, Variations des bacteriophage conditionees par les bacteries hostes. Ann. l'inst. Pastuer $84:$ 175-179. 